\title{
ALGEBRAIC MODELS FOR PROBABILITY MEASURES ASSOCIATED WITH STOCHASTIC PROCESSES
}

BY

\author{
B. M. SCHREIBER( $\left.{ }^{(}\right)$, T.-C. SUN AND A. T. BHARUCHA-REID ${ }^{(1)}$
}

\begin{abstract}
This paper initiates the study of probability measures corresponding to stochastic processes based on the Dinculeanu-Foiaș notion of algebraic models for probability measures. The main result is a general extension theorem of Kolmogorov type which can be summarized as follows: Let $\left\{\left(X, \mathscr{A}_{i}, \mu_{i}\right), i \in I\right\}$ be a directed family of probability measure spaces. Then there is an associated directed family of probability measure spaces $\left\{\left(G, \mathscr{B}_{i}, \nu_{i}\right), i \in I\right\}$ and a probability measure $\nu$ on the $\sigma$-algebra $\mathscr{B}$ generated by the $\mathscr{B}_{i}$ such that (i) $\nu(B)=\nu_{i}(B), B \in \mathscr{B}_{i}, i \in I$, and (ii) for each $i \in I$ the spaces $\left(X, \mathscr{A}_{i}, \mu_{i}\right)$ and $\left(G, \mathscr{B}_{i}, v_{i}\right)$ are conjugate. The importance of the main theorem is that under certain mild conditions there exists an embedding $\psi: X \rightarrow G$ such that the induced measures $\nu_{i}$ on $G$ are extendable to $\nu$, although the measures $\mu_{i}$ on $X$ may not be extendable. Using the algebraic model formulation, the Kolmogorov extension property and the notion of a representation of a directed family of probability measure spaces are discussed.
\end{abstract}

1. Introduction. Let $(\Omega, \mathscr{A}, \mu)$ be a probability measure space and $T$ a subset of the real line. If $(X, \mathscr{B})$ is some measurable space, then an $\mathscr{A}$-measurable function $x(\omega): \Omega \rightarrow X$ is called a random element in $X$. A random function, or stochastic process, defined on $T$ with values in $X$ is a function $x(t, \omega): T \times \Omega \rightarrow X$ such that $x(t, \omega)$ is a random element in $X$ for each $t \in T$. To fix ideas, we will restrict our attention to the classical case and take $X=R$ (the real line) and $\mathscr{B}$ the $\sigma$-algebra of Borel sets. Let $\mathfrak{X}$ denote the set of all functions $x=x(t)$ defined on $T=[a, b]$, say. Put

$$
C_{t}(B)=\{x: x(t) \in B\},
$$

where $t \in[a, b]$ and $B \in \mathscr{B}$. The sets $C_{t}(B)$ are called the one-dimensional cylinder sets of $\mathfrak{X}$, and finite intersections of the one-dimensional cylinder sets are simply referred to as cylinder sets. Let $\mathscr{F}$ denote the smallest $\sigma$-algebra containing all the cylinder sets. Put $\nu(F)=\mu \circ x^{-1}(F), F \in \mathscr{F}$. Then $\nu$ is a probability measure or distribution on $(\mathfrak{X}, \mathscr{F})$ corresponding to the stochastic process $x(t, \omega)$. Frequently, the probability measure space $(\mathfrak{X}, \mathscr{F}, \nu)$ itself is referred to as the stochastic process.

Received by the editors September 1, 1970.

AMS 1969 subject classifications. Primary 6005, 6040; Secondary 4250, 4254.

Key words and phrases. Stochastic processes, probability measures, algebraic models for probability measures, extension of probability measures, directed families of probability measure spaces, positive-definite functions, lifting of measure spaces.

(1) Research supported by National Science Foundation Grant No. GP-13741.

Copyright (C) 1971, American Mathematical Society 
In many applications the measurable space $(\mathfrak{X}, \mathscr{F})$ is not the appropriate space on which to construct a probability measure; and this leads to the construction and study of probability measures on complete separable metric spaces, various Banach spaces, etc. In particular, recent work on random equations leads to the study of random functions with values in a Banach space, and the study of probability measures on Banach spaces and other linear topological spaces.

In order to specify a stochastic process in the above measure-theoretic sense (i.e., to define $\nu$ on $\mathscr{F}$ ), it is sufficient to specify the probability measure on the cylinder sets; and values of $\nu$ on sets of the form $\bigcap_{i=1}^{n} C_{t_{i}}\left(B_{i}\right)$ are defined by its values on sets of the form $\bigcap_{i=1}^{n} C_{t_{i}}\left(\left(-\infty, \xi_{i}\right)\right)$, where the $\xi_{i}$ are arbitrary real numbers, and $\left\{t_{1}, t_{2}, \ldots, t_{n}\right\} \subset[a, b]$. Hence it is sufficient to specify the quantities

Put

$$
\nu\left(\bigcap_{i=1}^{n} C_{t_{i}}\left(\left(-\infty, \xi_{i}\right)\right)\right)
$$

$$
F_{t_{1}, \ldots, t_{n}}\left(\xi_{1}, \ldots, \xi_{n}\right)=\nu\left(\bigcap_{i=1}^{n} C_{t_{i}}\left(\left(-\infty, \xi_{i}\right)\right)\right)
$$

For any fixed values of $t_{1}, \ldots, t_{n}$, the function $F$ defined by (1.1) is a distribution function in the classical sense, and satisfies the following conditions:

(1) the same permutation applied to each of the sets $\left(t_{1}, \ldots, t_{n}\right)$ and $\left(\xi_{1}, \ldots, \xi_{n}\right)$ does not change the value of $F$;

(2) $\lim _{\xi_{n} \rightarrow \infty} F_{t_{1}, \ldots, t_{n}}\left(\xi_{1}, \ldots, \xi_{n}\right)=F_{t_{1}, \ldots, t_{n-1}}\left(\xi_{1}, \ldots, \xi_{n-1}\right)$.

Hence, in order to define $\nu$, it is sufficient to specify the distribution functions $F$ satisfying the above conditions. This set of functions is called the set of finitedimensional distributions of the stochastic process. Hence the definition of a probability measure on $(R, \mathscr{B})$ in classical probability theory, by means of a distribution function, is generalized in the theory of stochastic processes by means of the finite-dimensional distributions defined by (1.1). The fundamental Kolmogorov extension or consistency theorem [11] states that any set of distribution functions $F_{t_{1}}, \ldots, t_{n}\left(\xi_{1}, \ldots, \xi_{n}\right)$ which satisfies conditions (1) and (2) generates a probability measure $\nu$ and, therefore, defines a stochastic process. In addition to [11], we refer to Billingsley [2] and Neveu [14] for treatments of Kolmogorov's extension theorem.

In recent years developments in the theory of stochastic processes and its applications have led to the study of abstract space-valued stochastic processes; and the study of the probability measures associated with these processes has required generalizations of Kolmogorov's theorem. Needless to say, Kolmogorov's theorem has served as the prototype for these generalizations. The reader is referred to the work of Gel'fand and Vilenkin [6], Kirk [10], Minlos [13], Parthasarathy [15], Segal [19], Silov [20], and Spaček [21]. The use of inductive (or direct) and projective (or inverse) systems of probability measure spaces in the study of stochastic processes is due to Bochner [3]. Recently, Choksi [4], Métivier [12], Raoult [16], and Scheffer [18] have used the same approach in their studies. 
For a detailed exposition of projective limits of probability measure spaces, we refer to the forthcoming monograph of M. M. Rao entitled Stochastic processes.

In this paper we initiate the study of probability measures corresponding to stochastic processes utilizing the Dinculeanu-Foiaş notion of algebraic models for probability measures [5]. Briefly, given a probability measure space $(X, \mathscr{A}, \mu)$, an algebraic model for $\mu$ is a pair $(\Gamma, \varphi)$ consisting of an Abelian group $\Gamma$ and a positive-definite complex-valued function $\varphi$ on $\Gamma$. In $\$ 2$ we summarize the basic definitions and properties of algebraic models for measures and give some concrete examples of algebraic models for probability measures.

Because of the importance of extension or consistency theorems in the general theory of stochastic processes, in this paper we formulate an algebraic model approach to extension theorems, and prove, in \$3, a general extension theorem of Kolmogorov type. This result can be summarized as follows: Let $\left\{\left(X, \mathscr{A}_{i}, \mu_{i}\right), i \in I\right\}$ be a directed family of probability measure spaces. Then there is an associated directed family of probability measure spaces $\left\{\left(G, \mathscr{B}_{i}, \nu_{i}\right), i \in I\right\}$ and a probability measure $\nu$ on the $\sigma$-algebra $\mathscr{B}$ generated by the $\mathscr{B}_{i}$ such that (i) $\nu(B)=\nu_{i}(B), B \in \mathscr{B}_{i}$, $i \in I$, and (ii) for each $i \in I$ the spaces $\left(X, \mathscr{A}_{i}, \mu_{i}\right)$ and $\left(G, \mathscr{B}_{i}, \nu_{i}\right)$ are conjugate. The proof of this result, and subsequent results in $\$ 4$, utilizes the notions of inductive (or direct) and projective (or inverse) systems. We refer to Kelley and Namioka [9] for a discussion of inductive and projective systems.

The importance of our main theorem (Theorem 3) is that under certain mild conditions there exists an embedding $\psi: X \rightarrow G$ (it may not be 1-1) such that the induced measures $\nu_{i}$ on $G$ are extendable to a $\nu$ as above, although the measures $\mu_{i}$ on $X$ may not be extendable. This suggests that in those cases where the $\mu_{i}$ are not extendable on $X$, we may work with the $\nu_{i}$ on $G$ instead. As far as the stochastic processes are concerned, since the measures $\mu_{i}$ and $\nu_{i}$ are conjugate, the probability measure spaces $\left(X, \mathscr{A}_{i}, \mu_{i}\right)$ and $\left(G, \mathscr{B}_{i}, \nu_{i}\right)$ are, through the mapping $\psi$, indistinguishable. It is important to note that the enlarged spaces $\left(G, \mathscr{B}_{i}, \nu_{i}\right)$ have much nicer topological and algebraic properties than the basic spaces $\left(X, \mathscr{A}_{i}, \mu_{i}\right)$; indeed this may be one of the main advantages of utilizing algebraic models for the study of probability measures associated with stochastic processes.

The above remarks provide the motivation for the problems considered in $\$ 4$; namely, using the algebraic model formulation, we consider the Kolmogorov extension property and the notion of a representation of a directed family of probability measure spaces.

Finally, in $\$ 5$ we discuss two examples: (1) the embedding of a nonextendable system into an extendable system, and (2) a nonsequentially maximal system.

In subsequent papers we will consider (1) algebraic models for some concrete probability measures (e.g., Wiener measure, Gaussian measures, $L$-measures, etc.) which are of interest in the theory of stochastic processes and its applications, and (2) an algebraic model formulation of a transformation theory of probability measures under mappings of the spaces on which the measures are defined. 
2. Algebraic models for measures: Basic definitions and properties. In this section we collect for reference purposes several definitions and properties of algebraic models for measures, most of which appear in [5]. We also give some concrete examples of algbraic models for probability measures.

Let $(X, \mathscr{A}, \mu)$ be a probability measure space, and denote by $\Gamma(\mu)$ the set of (equivalence classes of) functions $\gamma \in L_{\infty}(\mu)$ with $|\gamma| \equiv 1 . \Gamma(\mu)$ is a multiplicative group with complex conjugate $\bar{\gamma}$ as the inverse of a function $\gamma \in \Gamma(\mu)$. If $(X, \mathscr{A}, \mu)$ is a complete probability measure space, then using the existence of a lifting (cf. A. Ionescu Tulcea and C. Ionescu Tulcea [8, Chapter IV]) we can consider $\Gamma(\mu)$ as a group of complex-valued $\mu$-measurable functions $\gamma$ on $X$ with $|\gamma(x)|=1$ a.e. such that $\gamma_{1}, \gamma_{2} \in \Gamma(\mu)$ and $\gamma_{1}=\gamma_{2}$ a.e. imply $\gamma_{1}(x)=\gamma_{2}(x)$ for all $x \in X$. It follows that we may also assume $|\gamma(x)|=1$ for all $x \in X, \gamma \in \Gamma(\mu)$, by replacing $\gamma$ by the function $\gamma^{\prime}$ such that

$$
\begin{array}{rlrl}
\gamma^{\prime}(x) & =e^{i \theta} & & \text { if } \gamma(x)=\rho e^{i \theta}, \rho \neq 0, \\
& =1 & \text { if } \gamma(x)=0 .
\end{array}
$$

Definition 1. A pair $(\Gamma, \varphi)$ consisting of an Abelian group $\Gamma$ and a positivedefinite complex-valued function $\varphi$ on $\Gamma$ such that $\varphi(\gamma)=1$ if and only if $\gamma=1$ is called an algebraic measure system (or simply a measure system).

Definition 2. A measure system $(\Gamma, \varphi)$ is said to be an algebraic model for the probability measure $\mu$ if there exists an injective homomorphism $h$ on $\Gamma$ into $\Gamma(\mu)$ such that (i) $h(\Gamma)$ generates $L_{2}(\mu)$, and (ii) $\varphi(\gamma)=\varphi_{\mu}(h(\gamma)), \gamma \in \Gamma$, where $\varphi_{\mu}(\lambda)=\int_{X} \lambda d \mu$.

We now give examples of algebraic models for some probability measures.

(1) One choice for an algebraic model for $\mu$ is $\left(\Gamma(\mu), \varphi_{\mu}\right)$. Another much smaller model is obtained as follows. Let $\mathscr{A}_{0}$ be any subalgebra of $\mathscr{A}$ which generates $\mathscr{A}$ as a $\sigma$-algebra, and for $A \in \mathscr{A}_{0}, x \in X$, set

$$
\begin{aligned}
\gamma_{A}^{\prime}(x) & =1, \quad x \in A, \\
& =-1, \quad x \notin A ;
\end{aligned}
$$

and let $\gamma_{A}$ be the unique function in $\Gamma(\mu)$ such that $\gamma_{A}=\gamma_{A}^{\prime}$ a.e. If $\Gamma_{0}=\left\{\gamma_{A} \mid A \in \mathscr{A}_{0}\right\}$, then $\left(\Gamma_{0}, \varphi_{\mu}\right)$ is an algebraic model for $\mu$, since $\frac{1}{2}\left(1+\gamma_{A}^{\prime}\right)=\chi_{A}$, where $\chi_{A}$ is the characteristic function of $A \in \mathscr{A}_{0}$. In particular, if $(X, \mathscr{A}, \mu)$ is separable, then $\Gamma_{0}$ may be chosen countable.

(2) Let $X$ be a Hausdorff topological space, $\mathscr{A}$ the completion of the Borel sets in $X$ with respect to the regular probability measure $\mu$ on $X$ (i.e., $\mu(A)$ $=\sup \{\mu(K): K$ compact, $K \subset A\}, A \in \mathscr{A})$ whose support is $X$. Then $\left(\Gamma, \varphi_{\mu}\right)$ is an algebraic model for $\mu$, where $\Gamma$ is the group of all continuous functions on $X$ of unit modulus.

(3) Let $X$ be a locally convex linear topological space with dual $X^{*}$, let $\mu$ be a cylinder measure (cf. Gel'fand and Vilenkin [6, p. 307]) on $X^{*}$, and let

$$
\hat{\mu}(x)=\int_{X^{*}} e^{i\left\langle x, x^{*}\right\rangle} d \mu\left(x^{*}\right)
$$


be the Fourier transform or characteristic functional of $\mu$ (cf. Gel'fand and Vilenkin [6, p. 260]). Let $K$ be the closed (additive) subgroup of $X$ of all $x$ such that $\hat{\mu}(x)=1$. If $\hat{\mu}$ also denotes the induced function on $X / K$, then $(X / K, \mu)$ is an algebraic model for $\mu$. Indeed, if $X$ is finite dimensional, then $\hat{\mu}$ is the ordinary FourierStieltjes transform; and the desired result follows from (4) given below. In the general case it suffices to notice, by looking at finite-dimensional subspaces of $X$, that the characteristic function of every cylinder set in $X^{*}$ is in the $L_{2}(\mu)$-span of the functions $e^{i\langle x, \cdot\rangle}, x \in X$.

(4) If $G$ is a locally compact Abelian group with character group $\Gamma$ and $\mu$ is a regular Borel probability measure on $G$ such that its support generates $G$, then $(\Gamma, \hat{\mu})$ is an algebraic model for $\mu$, where $\hat{\mu}$ is the Fourier-Stieltjes transform of $\mu$. (In this case the mapping $\psi$ defined in Lemma 1 below is just the embedding of $G$ in its Bohr compactification (Rudin [17, p. 30]).)

Proof. It is well known that $\hat{\mu}$ is positive-definite, and that if $\mu$ is not concentrated on any proper closed subgroup of $G$ then $\hat{\mu}=1$ only at $\gamma=1$ (Rudin [17, p. 53]). It remains to show that $\Gamma$ generates $L_{2}(\mu)$; and to do this it suffices to show that given $\varepsilon>0$ and $f$ continuous on $G$ with compact support, then there exists a trigonometric polynomial $P$ on $G$ with $\|f-P\|_{L_{2}(\mu)}<\varepsilon$. Thus, let $K$ be a compact subset of $G$ such that $f=0$ outside $K$ and $\mu\left(K^{C}\right)<\delta$ (to be prescribed). By embedding $G$ in its Bohr compactification $G^{B}$ and applying Tietze's theorem, $f$ can be extended to a continuous function $g$ on $G^{B}$ (i.e., an almost periodic function on $G$ ) with $\|g\|_{G^{B}}=\|g\|_{G}=\|f\|_{G}$. Since the trigonometric polynomials are dense in $C\left(G^{B}\right)$, we can find a trigonometric polynomial $P$ on $G^{B}$ (hence on $G$ ) with $\|g-P\|_{G}<\delta$. Then

$$
\begin{aligned}
\|f-P\|_{L_{2}(\mu)}^{2} & =\int_{K}|f-P|^{2} d \mu+\int_{K^{C}}|f-P|^{2} d \mu=\int_{K}|g-P|^{2} d \mu+\int_{K^{c}}|P|^{2} d \mu \\
& <\delta^{2} \mu(K)+\left(\delta+\|g\|_{G}\right) \mu\left(K^{C}\right)<\delta^{2}+\left(\delta+\|f\|_{G}\right) \delta=2 \delta^{2}+\|f\|_{G} \delta .
\end{aligned}
$$

Hence, prescribing $\delta$ sufficiently small gives $\|f-P\|_{L_{2}(\mu)}<\varepsilon$.

The last example we give is the following.

(5) Let $\left(X_{i}, \mathscr{A}_{i}, \mu_{i}\right)$ be probability measure spaces with algebraic models $\left(\Gamma_{i}, \varphi_{i}\right)$, with $\Gamma_{i}$ considered as a subgroup of $\Gamma\left(\mu_{i}\right), i=1,2$. Let $\mu$ be a probability measure on the measurable space $\left(X_{1} \times X_{2}, \mathscr{A}_{1} \times \mathscr{A}_{2}\right)$ such that $\mu\left(A \times X_{2}\right)=\mu_{1}(A), A \in \mathscr{A}_{1}$, and $\mu\left(X_{1} \times A\right)=\mu_{2}(A), A \in \mathscr{A}_{2}$. Set $\Gamma=\left\{\gamma\left(x_{1}, x_{2}\right)=\gamma_{1}\left(x_{1}\right) \gamma_{2}\left(x_{2}\right) \mid \gamma_{1} \in \Gamma_{1}, \gamma_{2} \in \Gamma_{2}\right\}$, and $K=\{\gamma \in \Gamma \mid \gamma=1 \mu$-a.e. $\}$. Then $(\Gamma / K, \varphi)$ is an algebraic model for $\mu$, where $\varphi(\gamma+K)=\varphi_{\mu}(\gamma), \gamma \in \Gamma$. In particular, if $\mu_{1} \times \mu_{2} \ll \mu$, then $\left(\Gamma, \varphi_{\mu}\right)$ is an algebraic model for $\mu$; and $\Gamma \cong\left(\Gamma_{1} \times \Gamma_{2}\right) / \Lambda$, where $\Lambda$ consists of all pairs $\left(\gamma_{1}, \gamma_{2}\right)$ of constants such that $\gamma_{1}=\bar{\gamma}_{2}$. For in this case, if $\gamma\left(x_{1}, x_{2}\right)=\gamma\left(x_{1}\right) \gamma\left(x_{2}\right)=1 \mu$-a.e., then $\gamma=1$ $\mu_{1} \times \mu_{2}$-a.e. But then

$$
1=\int_{X_{1} \times X_{2}} \gamma d\left(\mu_{1} \times \mu_{2}\right)=\int_{x_{1}} \gamma_{1} d \mu_{1} \int_{x_{2}} \gamma_{2} d \mu_{2},
$$

so each of the integrals on the right is of unit modulus. Thus $\gamma_{1}$ and $\gamma_{2}$ are constants a.e., hence everywhere by the linearity of the liftings. The rest is clear. 
Let $\left(\mathscr{B}_{1}, \mu_{1}\right)$ and $\left(\mathscr{B}_{2}, \mu_{2}\right)$ be the measure algebras associated with the probability measure spaces $\left(X_{1}, \mathscr{A}_{1}, \mu_{1}\right)$ and $\left(X_{2}, \mathscr{A}_{2}, \mu_{2}\right)$, respectively (cf. Halmos [7, §15]).

Definition 3. The probability measures $\mu_{1}$ and $\mu_{2}$ are called conjugate if there exists a measure-preserving isomorphism of the $\sigma$-algebra $\mathscr{B}_{1}$ onto the $\sigma$-algebra $\mathscr{B}_{2}$; or equivalently, if there exists a linear isometry $\Phi$ of $L_{2}\left(\mu_{1}\right)$ onto $L_{2}\left(\mu_{2}\right)$ satisfying (i) $\Phi\left(L_{\infty}\left(\mu_{1}\right)\right)=L_{\infty}\left(\mu_{2}\right)$, and (ii) $\Phi(f g)=\Phi(f) \Phi(g), f, g \in L_{\infty}\left(\mu_{1}\right)$. (It can be shown that $\Phi$ is then an $L_{\infty}$-isometry; cf. [5].)

Definition 4. Two measure systems $\left(\Gamma_{1}, \varphi_{1}\right)$ and $\left(\Gamma_{2}, \varphi_{2}\right)$ are said to be isomorphic if there exists an isomorphism $\psi$ of $\Gamma_{1}$ onto $\Gamma_{2}$ such that $\varphi_{2} \circ \psi=\varphi_{1}$.

THEOREM 1 (THEOREM 2 IN [5]). Two probability measures are conjugate if and only if they have isomorphic algebraic models.

THEOREM 2 (THEOREM 3 IN [5]). Every measure system is an algebraic model for a regular Borel probability measure on a compact Abelian group. More precisely, given a measure system $(\Gamma, \varphi)$ let $\Gamma$ be given the discrete topology, let $G$ denote the (compact) character group of $\Gamma$, and let $\mu$ be the (unique) regular Borel probability measure on $G$ whose Fourier-Stieltjes transform is $\varphi$. Then $(\Gamma, \varphi)$ is an algebraic model for $\mu$.

It follows that given the probability measure space $(X, \mathscr{A}, \mu)$ and an algebraic model $(\Gamma, \varphi)$ for $\mu$ (which we can consider as a subsystem of $\left(\Gamma(\mu), \varphi_{\mu}\right)$ ), there is a Borel measure $\nu$ on the character group $G$ of $\Gamma$ such that $\mu$ and $\nu$ are conjugate. In fact, the conjugation is given by a mapping of $X$ into $G$ :

Lemma 1. Let $(X, \mathscr{A}, \mu), G, \Gamma$, and $\nu$ be as above. Then there is a measurable mapping $\psi: X \rightarrow G$ such that

$$
\Phi(f) \circ \psi=f \quad \mu \text {-a.e., } \quad f \in L_{2}(\mu),
$$

where $\Phi$ is as in Definition 3.

Proof. $\Phi$ is obtained by linearly extending the map $(g, \Phi(\gamma))=(g, \gamma)$. (Here $(\cdot, \cdot)$ denotes the pairing of $G$ and $\Gamma$ via Pontrjagin duality (cf. Rudin [17, p. 28]).) Thus define $\psi$ by $(\psi(x), \gamma)=\gamma(x), x \in X, \gamma \in \Gamma$. Then (2.1) holds everywhere on $X$ for $f=\gamma \in \Gamma$, and hence on the linear span of $\Gamma$. Since the linear span is dense in $L_{2}(\mu)$, the result follows upon taking limits.

In particular, if $A \in \mathscr{A}$, then $\Phi\left(\chi_{A}\right)$ is the characteristic function of an open and closed subset $E$ of $G$ (since $\Phi^{2}\left(\chi_{A}\right)=\Phi\left(\chi_{A}\right)$ and $\Phi: L_{\infty}(\mu) \rightarrow C(G)$ ) such that $\mu\left(A \Delta \psi^{-1}(E)\right)=0$. (Note that $\psi^{-1}(E) \in \mathscr{A}$ since $\mu$ is complete, and trigonometric polynomials are dense in $C(G)$.)

We conclude these remarks by pointing out that the fact that one can always choose an algebraic model $(\Gamma, \varphi)$ for any probability space $(X, \mathscr{A}, \mu)$ such that $\Gamma$ is a group of measurable functions does not depend on the existence of liftings. Indeed, let $\Gamma^{*}$ denote the collection of all $\mathscr{A}$-measurable functions of unit modulus, 
and set $K=\left\{\gamma \in \Gamma^{*} \mid \gamma=1\right.$ a.e. $\}$. Let $\mathscr{R}$ denote the collection of all subgroups $\Gamma$ of $\Gamma^{*}$ such that $\Gamma \cap K=\{1\}$.

LEMMA 2. Given any $\Gamma_{0} \in \mathscr{R}$ there exists $a \Gamma \in \mathscr{R}$ such that $\Gamma_{0} \subset \Gamma$ and $\Gamma$ generates $L_{2}(\mu)$. Hence $\left(\Gamma, \varphi_{\mu}\right)$ is an algebraic model for $\mu$. In particular, if $\mathscr{B}$ is a complete $\sigma$-subalgebra of $\mathscr{A}$, and setting $\nu=\mu \mid \mathscr{B},\left(\Gamma_{0}, \varphi_{v}\right)$ is an algebraic model for $\nu$, then $\Gamma_{0}$ can be extended to a subgroup $\Gamma$ of $\Gamma^{*}$ such that $\left(\Gamma, \varphi_{\mu}\right)$ is an algebraic model for $\mu$.

Proof. Let $\mathscr{R}_{0}=\left\{\Gamma \in \mathscr{R} \mid \Gamma \supset \Gamma_{0}\right\}$, and let $\mathscr{R}_{0}$ be partially ordered by inclusion. Since the union of the groups in any chain in $\mathscr{R}$ is in $\mathscr{R}$, we may choose by Zorn's lemma a maximal element $\Gamma$ of $\mathscr{R}_{0}$ (which will then be maximal in $\mathscr{R}$ ). To show that $\Gamma$ generates $L_{2}(\mu)$, let $A \in \mathscr{A}$ and define $\gamma_{A}^{\prime}$ as in Example 1 above. Since $\left(\chi_{A}^{\prime}\right)^{2}=1$, it follows from the maximality that $\gamma_{A}^{\prime} \in \Gamma K$; so $\Gamma$ generates $L_{2}(\mu)$ as in Example 1.

3. The main theorem. Throughout this section all probability measure spaces are assumed complete.

Definition 5. A family $\left\{\left(X, \mathscr{A}_{i}, \mu_{i}\right), i \in I\right\}$ of probability measure spaces will be called a directed family if $(I, \leqq)$ is a directed set and the following conditions are satisfied: (i) $\mathscr{A}_{i} \subset \mathscr{A}_{j}, i \leqq j$, (ii) $\mu_{i}(A)=\mu_{j}(A), A \in \mathscr{A}_{i}, i \leqq j$.

We now state and prove the main theorem of this paper.

TheOREM 3. Let $\left\{\left(X, \mathscr{A}_{i}, \mu_{i}\right), i \in I\right\}$ be a directed family of probability measure spaces. Then there is a directed family $\left\{\left(G, \mathscr{B}_{i}, \nu_{i}\right), i \in I\right\}$ of probability measure spaces over a compact Abelian group $G$ and a probability measure $\nu$ on the $\sigma$-algebra $\mathscr{B}$ generated by the $\mathscr{B}_{i}$ such that

(i) $\nu(B)=\nu_{i}(B), B \in \mathscr{B}_{i}, i \in I$.

(ii) For each $i \in I$, the spaces $\left(X, \mathscr{A}_{i}, \mu_{i}\right)$ and $\left(G, \mathscr{B}_{i}, \nu_{i}\right)$ are conjugate; in fact, there exists a mapping $\psi_{i}: X \rightarrow G$ satisfying

(1) $\psi_{i}^{-1}(B) \in \mathscr{A}_{i}, B \in \mathscr{B}_{i}, i \in I$.

(2) Given $A \in \mathscr{A}_{i}$, there exists a $B \in \mathscr{B}_{i}$ such that $\nu_{i}(B)=\mu_{i}(A)$ and $\mu_{i}\left(A \Delta \psi_{i}^{-1}(B)\right)$ $=0$.

Proof. For each $i \in I$ let $\Gamma_{i}$ denote the group $\Gamma\left(\mu_{i}\right)$ of complex-valued $\mathscr{A}_{i}$ measurable functions of constant modulus 1 on $X$ (cf. §2); and define $\varphi_{i}(\gamma)$ $=\int_{X} \gamma d \mu_{i}, \gamma \in \Gamma_{i}, i \in I$. Thus $\left(\Gamma_{i}, \varphi_{i}\right)$ is an algebraic model for $\mu_{i}$; and if $i \leqq j$ it follows from Definition 5 that there is a natural isomorphism $\varepsilon_{i j}$ of $\Gamma_{i}$ into $\Gamma_{j}$ such that

$$
\begin{array}{lll}
\varepsilon_{i j}(\gamma)=\gamma \quad \mu_{j} \text {-a.e., } & & \gamma \in \Gamma_{i}, \\
\varphi_{i}(\gamma)=\varphi_{j}\left(\varepsilon_{i j}(\gamma)\right), & \gamma \in \Gamma_{i} .
\end{array}
$$

Indeed, $\varepsilon_{i j}$ is uniquely determined by (3.1) as a homomorphism satisfying (3.2); and if $\varepsilon_{i j}(\gamma)=1$ then $\gamma=1 \mu_{j}$-a.e., whence $\mu_{i}$-a.e. by the compatability condition (ii) of Definition 5, so $\gamma=1$ and $\varepsilon_{i j}$ is an isomorphism. It also follows from (3.1) that the $\Gamma_{i}$ and $\varepsilon_{i j}$ satisfy the axioms of an inductive limit system; that is $\varepsilon_{i i}$ $=\operatorname{Id}\left(\Gamma_{i}\right), i \in I$, and $\varepsilon_{j k} \varepsilon_{i j}=\varepsilon_{i k}, i \leqq j \leqq k$. 
Let $\Gamma$ denote the inductive-limit group $\Gamma=$ ind $\lim _{i} \Gamma_{i}$. Thus for each $i \in I$ there is an isomorphism $\varepsilon_{i}$ of $\Gamma_{i}$ into $\Gamma$; and we have

$$
\begin{gathered}
\Gamma=\bigcup_{i \in I} \varepsilon_{i}\left(\Gamma_{i}\right), \\
\varepsilon_{j} \varepsilon_{i j}=\varepsilon_{i}, \quad i \leqq j .
\end{gathered}
$$

Let $\varphi$ be the function on $\Gamma$ defined by

$$
\varphi \varepsilon_{i}=\varphi_{i}, \quad i \in I .
$$

It follows from (3.2)-(3.5) that $\varphi$ is a well-defined positive-definite function on $\Gamma$ and that $\varphi(\gamma)=1$ implies $\gamma=1$. That is, $(\Gamma, \varphi)$ is a measure system (Definition 1). Let $G_{i}, \lambda_{i}$ (respectively, $G, \nu$ ) be as in Theorem 2 for $\left(\Gamma_{i}, \varphi_{i}\right), i \in I$ (respectively, $(\Gamma, \varphi)$ ). Thus $G$ is the projective limit $G=\operatorname{proj} \lim _{i} G_{i}$ of the compact groups $G_{i}$, $i \in I$, with respect to the adjoint homomorphisms $\varepsilon_{i}^{*}: G \rightarrow G_{i}$ and $\varepsilon_{i j}^{*}: G_{j} \rightarrow G_{i}$ of the $\varepsilon_{i}$ and $\varepsilon_{i j}$, respectively.

If $\mathscr{B}\left(G_{i}\right)$ denotes the $\sigma$-algebra of $\lambda_{i}$-measurable subsets of $G_{i}$, then putting $\mathscr{B}_{i}=\varepsilon_{i}^{*-1}\left(\mathscr{B}\left(G_{i}\right)\right)$ and $\nu_{i}=\lambda_{i} \circ \varepsilon_{i}^{*}$, (i) of the theorem follows from (3.5) and the fact that the $\Gamma_{i}$ (respectively, $\Gamma$ ), considered as functions on $G$, generate $L_{2}\left(\nu_{i}\right)$ (respectively, $\left.L_{2}(v)\right)$. For $x \in X$, let $\psi_{i}(x) \in G$ be any element of $G$ satisfying

$$
\left(\psi_{i}(x), \varepsilon_{i}(\gamma)\right)=\gamma(x), \quad \gamma \in \Gamma_{i}, i \in I .
$$

(Here $(\cdot, \cdot)$ is as in the proof of Lemma $1, \S 2$.) Now, (ii) of the theorem follows from Lemma 1 and the observation that if the mappings $\psi^{i}: X \rightarrow G_{i}, i \in I$, are as in $\S 2$, then $\psi^{i}=\varepsilon_{i}^{*} \circ \psi_{i}, i \in I$.

REMARKS. (1) The fact that the $\Gamma_{i}$ were chosen as groups of functions was used only to construct the mappings $\psi_{i}$; the remainder of the proof of Theorem 3 does not depend on the existence of a lifting, as the mappings (3.1) are easily seen to be well defined on the groups of equivalence classes.

(2) The choice of $\Gamma_{i}=\Gamma\left(\mu_{i}\right)$ and $\varphi_{i}, i \in I$, could, of course, be replaced by any algebraic models for the $\mu_{i}$ such that the $\Gamma_{i}$ form an inductive system with respect to some $\varepsilon_{i j}$ for which (3.2) holds. Moreover, if the groups can be chosen so that (3.1) holds everywhere, then for each $x \in X$ there is a unique element $\psi(x) \in G$ for which (3.6) holds with $\psi_{i}(x)=\psi(x)$, all $i \in I$, and we can set all $\psi_{i}=\psi$ in the theorem.

4. The Kolmogorov extension property. Theorem 3 can be applied to an inverse system of probability spaces $\left\{\left(X_{i}, \mathscr{A}_{i}, \mu_{i}\right), i \in I\right\}$, related by measurable, measure-preserving mappings $f_{i j}$, by setting $X=$ proj $\lim _{i} X_{i}$ (set-theoretic projective limit) and considering the directed system $\left\{\left(X, \mathscr{A}_{i}^{*}, \mu_{i}^{*}\right), i \in I\right\}$ induced by the natural mappings $f_{i}: X \rightarrow X_{i}, i \in I$. In probability theory $I$ is usually assumed to be all finite subsets $F$ of a set $S$, and a fundamental problem is that of determining whether there is a probability measure on the $\sigma$-algebra of subsets of $X=$ proj $\lim _{F} X_{F}=\prod_{s \in S} X_{\{s\}}$ generated by all the $\mathscr{A}_{F}^{*}$ (the cylinder sets) which extends all the probability measures $\mu_{F}^{*}$. 
The above considerations lead us to the following definitions:

Definition 6. A directed family of probability measure spaces $\left\{\left(X, \mathscr{A}_{i}, \mu_{i}\right), i \in I\right\}$ is said to have the Kolmogorov extension property (or simply, to be extendable) if there is a measure $\mu$ on the complete $\sigma$-algebra $\mathscr{A}$ generated by $\bigcup_{i \in I} \mathscr{A}_{i}$ such that $\mu=\mu_{i}$ on $\mathscr{A}_{i}, i \in I$.

Definition 7. A mapping $\psi: X \rightarrow G$, where $G$ is a compact Abelian group with character group $\Gamma$, is said to be a representation of a directed family of probability spaces $\left\{\left(X, \mathscr{A}_{i}, \mu_{i}\right), i \in I\right\}$ if

(i) for each $\gamma \in \Gamma, \gamma \circ \psi$ is $\mathscr{A}_{i}$-measurable for some $i \in I$;

(ii) $\left(\Gamma_{i}, \varphi_{i}\right)$ is an algebraic model for $\mu_{i}, i \in I$, where $\Gamma_{i}=\left\{\gamma \in \Gamma \mid \gamma \circ \psi\right.$ is $\mathscr{A}_{i^{-}}$ measurable $\}$ and $\varphi_{i}(\gamma)=\int_{X}(\gamma \circ \psi) d \mu_{i}, \gamma \in \Gamma_{i}$.

In view of the above, a directed family of probability measure spaces possesses a representation if and only if $G$ is as in Theorem 3 and the mappings $\psi_{i}$ which appear in the conclusion of that theorem can all be taken equal to $\psi$.

In the next theorem, we shall give a necessary and sufficient condition for a directed family of probability measures having a representation to be extendable. In the following we shall always use the notation of $\S 3$ and Theorem 3.

THEOREM 4. Let $\psi: X \rightarrow G$ be a representation of the directed family of probability measure spaces $\left\{\left(X, \mathscr{A}_{i}, \mu_{i}\right), i \in I\right\}$. Then the family has the Kolmogorov extension property if and only if $\nu^{*}(\psi(X))=1$ (where $\nu^{*}$ denotes outer measure).

Proof. Suppose the family is extendable. Then $\Gamma=$ ind $\lim _{i} \Gamma_{i}$; and if $\varphi$ is as defined from the $\varphi_{i}$ of Definition 7 as in (3.5), then $(\Gamma, \varphi)$ is an algebraic model for $\mu$. It now follows from Lemma 1 that $\nu^{*}(\psi(X))=1$. Conversely, if $\nu^{*}(\psi(X))=1$, we take $\mu_{0}$ to be the measure on $X$ given by $\mu_{0}\left(\psi^{-1}(E)\right)=\nu(E), E \in \mathscr{B}$. The completion $\mu$ of $\mu_{0}$ is then a probability measure on $\mathscr{A}$ such that $\mu=\mu_{i}$ on $\mathscr{A}_{i}, i \in I$.

Following Bochner [3, Chapter 5], who introduced the notion of a sequentially maximal family, we introduce the analogous definition for representations.

Definition 8. A representation $\psi: X \rightarrow G$ is said to be sequentially maximal if given $i_{1} \leqq i_{2} \leqq \cdots \leqq i_{n} \leqq \cdots$ in $I$ and

$$
g \in \bigcap_{n=1}^{\infty}\left(\varepsilon_{i_{n}}^{*}\right)^{-1} \circ \psi_{i_{n}}(X),
$$

where $\psi_{i}=\varepsilon_{i}^{*} \circ \psi, i \in I$, there exists an $x \in X$ such that $\psi_{i_{n}}(x)=\varepsilon_{i_{n}}^{*}(g), n=1,2, \ldots$

We have the following result:

COROLlaRY 1. Let $\psi$ be a sequentially maximal representation of the family $\left\{\left(X, \mathscr{A}_{i}, \mu_{i}\right), i \in I\right\}$ with the property that $\psi_{i}(X) \in \mathscr{B}\left(G_{i}\right), i \in I$. Then the family has the Kolmogorov extension property.

Proof. By Theorem 4 we must show, given $B \in \mathscr{B}$, with $\psi(X) \subset B$ that $\nu(B)=1$. Since every element of $\mathscr{B}$ is in the $\sigma$-algebra generated by countably many of the $\mathscr{B}_{i}$, we can find $i_{1} \leqq i_{2} \leqq \cdots$ such that $B$ is in the $\sigma$-algebra generated by $\bigcup_{n} \mathscr{B}_{i_{n}}$. We now replace $i_{n}$ by $n$, and put $\Gamma_{\infty}=\bigcup_{n} \Gamma_{n}$. Also, let $G_{\infty}$ be the dual of $\Gamma_{\infty}$, let 
$\pi_{\infty}: G \rightarrow G_{\infty}$ be the natural map, and let $\nu_{\infty}$ be as in Theorem 2 for $\left(\Gamma_{\infty}, \varphi \mid \Gamma_{\infty}\right)$. Then $\pi_{\infty}^{-1} \pi_{\infty}(B)=B, \nu_{\infty}\left(\pi_{\infty}(B)\right)=\nu(B)$, and $\psi_{\infty}=\pi_{\infty} \circ \psi: X \rightarrow G_{\infty}$ is a representation of $\left\{\left(X, \mathscr{A}_{n}, \mu_{n}\right), n \in N\right\}$. Let $\pi_{n}: G_{\infty} \rightarrow G_{n}$ be defined by $\varepsilon_{n}^{*}=\pi_{n} \circ \pi_{\infty}, n=1,2, \ldots$, and let $\psi^{n}: X \rightarrow G_{n}$ be as in Lemma 1 . Then by the sequential maximality,

$$
\bigcap_{n=1}^{\infty} \pi_{n}^{-1}\left(\psi^{n}(X)\right)=\psi_{\infty}(X) \subset \pi_{\infty}(B)
$$

Since, for each $n, \nu_{\infty}\left(\pi_{n}^{-1}\left(\psi^{n}(X)\right)\right)=\nu_{n}\left(\psi^{n}(X)\right)=1$, we have $\nu(B)=\nu_{\infty}\left(\pi_{\infty}(B)\right)=1$.

Another corollary is the following result due to Bochner [3, Theorem 5.1.1].

Corollary 2. Let $\left\{\left(X_{i}, \mathscr{A}_{i}, \mu_{i}\right), i \in I\right\}$ be an inverse system of Hausdorff topological measure spaces (in the sense of Example 2 of \$2) with respect to continuous mappings $f_{i j}: X_{j} \rightarrow X_{i}$. If the system is sequentially maximal, the induced directed family $\left\{\left(X, \mathscr{A}_{i}^{*}, \mu_{i}^{*}\right), i \in I\right\}$ has the Kolmogorov extension property.

Proof. Let $\left(\Gamma_{i}, \varphi_{\mu_{i}}\right)$ be as in Example 2 of $\S 2$, and let $\left(\Gamma_{i}^{*}, \varphi_{i}^{*}\right)$ be the induced algebraic model for $\mu_{i}^{*}$, where $\Gamma_{i}^{*}$ consists of functions on $X$. We recall that $\Gamma_{i}$ determines the topology on $G_{i}$; hence $\psi_{i}: X_{i} \rightarrow G_{i}$ (as in Lemma 1) is continuous. Also, the regularity of $\mu_{i}$ implies that $X_{i}$ contains a $\sigma$-compact subset of measure one. Therefore, $\psi_{i}\left(X_{i}\right) \in \mathscr{B}\left(G_{i}\right), i \in I$. Since $\Gamma_{i}^{*} \subset \Gamma_{j}^{*}$ if $i \leqq j$, there is a representation $\psi: X \rightarrow G$ of the directed family (where $G$ is the dual of $\Gamma=\bigcup_{i \in I} \Gamma_{i}^{*}=$ ind $\lim \Gamma_{i}$ ), such that $\psi_{i} \circ f_{i}=\varepsilon_{i}^{*} \circ \psi$. The sequential maximality of the given system and the fact that each $\psi_{i}$ is one-to-one then imply that the representation is sequentially maximal; and desired result follows from Corollary 1.

Though not every directed family of probability measure spaces possesses a representation, we can still obtain a necessary and sufficient condition for the family to have the Kolmogorov extension property by considering all increasing sequences in the indexing set $I$.

THeOREM 5. Let $\left\{\left(X, \mathscr{A}_{i}, \mu_{i}\right), i \in I\right\}$ be a directed family of probability measure spaces. The following are equivalent:

(i) The given family has the Kolmogorov extension property.

(ii) If $J$ is any directed subset of $I$ and $\psi: X \rightarrow G$ is a representation of the directed family $\left\{\left(X, \mathscr{A}_{i}, \mu_{i}\right), i \in J\right\}$, then $\nu^{*}(\psi(X))=1$.

(iii) If $J$ is any increasing sequence in I there is a representation of $\left\{\left(X, \mathscr{A}_{i}, \mu_{i}\right), i \in J\right\}$ such that $\nu^{*}(\psi(X))=1$.

Proof. Clearly (i) $\Rightarrow$ (ii). The given family is extendable if and only if every subfamily corresponding to an increasing sequence in $I$ is extendable (cf. the proof of Corollary 1). Thus the statement of the theorem will follow from Theorem 4 if we can show that, for $J$ an increasing sequence, the corresponding family has a representation. Lemma 2 implies that this is in fact the case.

REMARK. If $I$ is countable, the argument above shows that $\left\{\left(X, \mathscr{A}_{i}, \mu_{i}\right), i \in I\right\}$ always has a representation. Indeed, we may pick an increasing sequence $J$ in $I$ such that each element of $I$ is dominated by an element of $J$. Then, using Lemma 2 , 
Theorem 3, and Remark 2 which follows Theorem 3, it is easy to construct the desired representation. If $I$ is uncountable, this procedure fails. For example, if $I$ consists of all finite subsets of an uncountable set, then every chain in $I$ must be countable.

5. Some examples. In this section we discuss two examples:

(1) the embedding of a nonextendable system into an extendable one, and

(2) a nonsequentially maximal system.

(1) Consider the interval $[0,1)$ with Lebesgue measure $\xi$ on its Borel algebra of subsets $\mathscr{B}$. Let $V_{1}, V_{2}, \ldots$ be the Vitali nonmeasurable sets of $[0,1)$, and let $Y_{n}=\bigcup_{i=n}^{\infty} V_{i}$. Then (i) $Y_{n} \downarrow \varnothing$ as $n \rightarrow \infty$, and (ii) the outer measure $\xi^{*}\left(Y_{n}\right)=1$ for all $n$ (see Neveu [14, p. 84]). Now, for each $n$, put $X_{n}=\prod_{k=1}^{n} Y_{k}$, and let $\mathscr{A}_{n}$ be the $\sigma$-algebra of subsets of $X_{n}$ generated by $Y_{k} \cap \mathscr{B}, k=1,2, \ldots, n$. Define $\lambda_{n}$ on $\left(Y_{n}, Y_{n} \cap \mathscr{B}\right)$ by $\lambda_{n}\left(Y_{n} \cap A\right)=\xi(A)$ for each $A \in \mathscr{B}$; and define $\mu_{n}$ on $\left(X_{n}, \mathscr{A}_{n}\right)$ by $\mu_{n}=\lambda_{n} \circ \theta_{n}^{-1}$, where $\theta_{n}: Y_{n} \rightarrow X_{n}, \theta_{n}(x)=(x, x, \ldots, x), x \in Y_{n}$. (Note: $\theta_{n}^{-1}(E)=\varnothing$ if $E$ contains no diagonal elements.) Now, for $i<j$ consider the projection mapping $f_{i j}: X_{j} \rightarrow X_{i}$. Then $\left\{\left(X_{n}, \mathscr{A}_{n}, \mu_{n}\right), n=1,2, \ldots\right\}$, together with $\left\{f_{i j}\right\}$, is an inverse system of probability measure spaces; and it is easy to see that the inverse limit can be identified with $X=\prod_{k=1}^{\infty} Y_{k}$.

The family $\left\{\left(X_{n}, \mathscr{A}_{n}, \mu_{n}\right), n=1,2, \ldots\right\}$ is not extendable since the measurable diagonals $\Delta_{n}=\left\{x \in X \mid x_{1}=x_{2}=\cdots=x_{n}\right\}$ all have measure one, yet $\Delta_{n} \downarrow \varnothing$. This example is due to Andersen and Jessen [1]. We remark that the family is, however, sequentially maximal.

Now, for $n=1,2, \ldots$, let $T_{n}=[0,1), G_{n}=\prod_{k=1}^{n} T_{k}$, and $G=\prod_{k=1}^{\infty} T_{k}$. Let $\mathscr{B}_{n}$ be the product Borel algebra in $G_{n}$, and let $\mathscr{B}$ be the Borel algebra generated by all the $\mathscr{B}_{n}$ in $G$. Let $\theta_{n}$ be analogously extended to all of the $T_{n}$, and define $\nu_{n}$ on $\left(G_{n}, \mathscr{B}_{n}\right)$ by $\nu_{n}=\xi \circ \theta_{n}^{-1}$. Then the family $\left\{\left(G_{n}, \mathscr{B}_{n}, \nu_{n}\right), n=1,2, \ldots\right\}$ is extendable; in fact, the extended measure $\nu$ is concentrated on the diagonal

$$
\Delta=\left\{\left(x_{1}, x_{2}, \ldots\right) \in G \mid x_{1}=x_{2}=\cdots\right\} .
$$

Let us now embed each $X_{n}$ into $G_{n}$ by the mapping $\psi_{n}(x)=x, x \in X_{n}$. Then, for each $n,\left(X_{n}, \mathscr{A}_{n}, \mu_{n}\right)$ and $\left(G_{n}, \mathscr{B}_{n}, \nu_{n}\right)$ are conjugate; so we have given a concrete construction of the type considered in Theorem 3 . We could have obtained exactly the same construction by the method used to prove Theorem 3 if we had begun with the groups $\Gamma_{k}$ of functions on $Y_{k}$ of the form $\gamma_{m}(x)=e^{i m x}, m=0, \pm 1, \pm 2, \ldots$

(2) Let $X_{i}$ be the additive group of integers modulo $2^{i}, i=1,2, \ldots$, and let $X_{\omega}$ be the set of all nonnegative integers. Define, for $i<j$ ( $j$ could be $\omega$ ), $f_{i j}: X_{j} \rightarrow X_{i}$ such that $f_{i j}(x)=y$, where $y \equiv x\left(\bmod 2^{i}\right)$; and choose any probability measure $\mu_{\omega}$ on $X_{\omega}$, and, for each $i=1,2, \ldots$, let $\mu_{i}=\mu_{\omega} \circ f_{\omega i}^{-1}$. The family $\left\{X_{i}, i=1,2, \ldots, \omega\right\}$ together with $\left\{f_{i j}\right\}$ and $\left\{\mu_{i}\right\}_{i=1}^{\omega}$ form an inverse system of probability measure spaces, and since the index set in this case has a largest element, namely $\omega$, the inverse limit system is equivalent to just $\left(X_{\omega}, \mu_{\omega}\right)$ and thus is extendable. 
We remark that the family $\left\{X_{i}, i=1,2, \ldots, \omega\right\}$ is not sequentially maximal. Consider the sequence $x_{i}^{0}=2^{i}-1 \in X_{i}, i=1,2, \ldots$ There exists no $x \in X_{\infty}$ $=\left\{\left\{x_{i}\right\}_{i=1}^{\omega} \mid f_{i j}\left(x_{j}\right)=x_{i}, i<j\right\}$ (the inverse limit of $\left\{X_{i}\right\}$ ) such that $x_{i}=x_{i}^{0}$ for all $i=1,2, \ldots$

The above examples seem to indicate that the condition of sequential maximality is independent of the problem of extending probability measures associated with stochastic processes, and its use is just a convenience in the proofs of extension theorems.

\section{REFERENCES}

1. E. S. Andersen and B. Jessen, On the introduction of measures in infinite product sets, Danske Vid. Selsk. Mat.-Fys. Medd. 25 (1948). MR 10, 239.

2. P. Billingsley, Convergence of probability measures, Wiley, New York, 1968. MR 38 \#1718.

3. S. Bochner, Harmonic analysis and the theory of probability, Univ. of California Press, Berkeley, 1955. MR 17, 273.

4. J. R. Choksi, Inverse limits of measure spaces, Proc. London Math. Soc. (3) 8 (1958), 321-342. MR 20 \#3251.

5. N. Dinculeanu and C. Foiaș, Algebraic models for measures, Illinois J. Math. 12 (1968), 340-351. MR 37 \#1548.

6. I. M. Gel'fand and N. Ja. Vilenkin, Generalized functions. Vol. 4, Some applications of harmonic analysis, Fizmatgiz, Moscow, 1961; English transl., Academic Press, New York, 1964. MR 26 \#4173; MR 30 \#4152.

7. P. R. Halmos, Lectures on Boolean algebras, Van Nostrand Math. Studies, no. 1, Van Nostrand, Princeton, N. J., 1963. MR 29 \#4713.

8. A. Ionescu Tulcea and C. Ionescu Tulcea, Topics in the theory of liftings, Springer-Verlag, New York, 1969.

9. J. L. Kelley and I. Namioka, Linear topological spaces, University Series in Higher Math., Van Nostrand, Princeton, N. J., 1963. MR 29 \#3851.

10. R. B. Kirk, Kolmogorov type consistency theorems for products of locally compact, B-compact spaces, Nederl. Akad. Wetensch. Proc. Ser. A $73=$ Indag. Math. 32 (1970), 77-81.

11. A. N. Kolmogorov, Grundbegriffe der Wahrscheinlichkeitsrechnung, Springer, Berlin, 1933; English transl., Chelsea, New York, 1956. MR 18, 155.

12. M. Métivier, Limites projectives de mesures. Martingales. Applications, Ann. Mat. Pura Appl. (4) 63 (1963), 225-352. MR 29 \#212.

13. R. A. Minlos, Generalized random processes and their extension to a measure, Trudy Moskov, Mat. Obšč. 8 (1959), 497-518; English transl., Selected Transl. Math. Statist. and Prob., vol. 3, Amer. Math. Soc., Providence, R. I., 1963, pp. 291-313. MR 21 \#7563; MR 27 \#4266.

14. J. Neveu, Bases mathématiques du calcul des probabilités, Masson, Paris, 1964; English transl., Holden-Day, San Francisco, Calif., 1965. MR 33 \#6659; 6660.

15. K. R. Parthasarathy, Probability measures on metric spaces, Probability and Math. Statist., no. 3, Academic Press, New York, 1967. MR 37 \#2271.

16. J.-P. Raoult, Limites projectives de mesures $\sigma$-finies et probabilités conditionnelles, C.R. Acad. Sci. Paris 260 (1965), 4893-4896. MR 32 \#478.

17. W. Rudin, Fourier analysis on groups, Interscience Tracts in Pure and Appl. Math., no. 12, Interscience, New York, 1962. MR 27 \#2808.

18. C. L. Scheffer, Limits of directed projective systems of probability spaces, Z. Wahrscheinlichkeitstheorie und Verw. Gebiete 13 (1969), 60-80. 
19. I. E. Segal, Abstract probability spaces and a theorem of Kolmogoroff, Amer. J. Math. 76 (1954), 721-732. MR 16, 149.

20. G. E. Silov, On measures in linear spaces, Dokl. Akad. Nauk SSSR 169 (1966), 46-48 =Soviet Math. Dokl. 7 (1966), 884-887. MR 35 \#1746.

21. A. Spaček, Probability measures in infinite Cartesian products, Illinois J. Math. 4 (1960), 210-220. MR 22 \#12547.

Wayne State University,

Detroit, Michigan 48202 